\title{
Understanding Textbook of History with Pictorial Representation using NLP
}

\author{
Abhishek Mukherjee \\ Department of Computer \\ Engineering \\ Sinhgad Institute of Technology \\ and Science \\ 49/1, Opp. Mumbai Bengaluru \\ Westerly Bypass, Narhe, \\ Pune, India \\ Pin No.: 411041
}

\author{
Nikhar Jain \\ Department of Computer \\ Engineering \\ Sinhgad Institute of Technology \\ and Science \\ 49/1, Opp. Mumbai Bengaluru \\ Westerly Bypass, Narhe, \\ Pune, India \\ Pin No.: 411041
}

\author{
Pratik Nandurkar \\ Department of Computer \\ Engineering \\ Sinhgad Institute of Technology \\ and Science \\ 49/1, Opp. Mumbai Bengaluru \\ Westerly Bypass, Narhe, \\ Pune, India \\ Pin No.: 411041
}

\author{
Pooja Vengurlekar \\ Asst. Prof \\ Department of Computer Engineering \\ Sinhgad Institute of Technology and Science \\ 49/1, Opp. Mumbai Bengaluru Westerly Bypass, Narhe, \\ Pune, India \\ Pin No.: 411041
}

\begin{abstract}
Every day the number data is generated on the internet. It is very challenging to determine the importance of the content. Text picturing helps a lot to understand the concept related to the text. Text picturing is a cognitive aid that can help with text understanding, as it helps users decide if the text deserves closer look by showing relevant pictures along with the text. The writers, bloggers, and editorial person link the text with media content is crucial. Visual content helps readers follow the root of a discussion or identify the core theme of an article. In efforts to arrange and display helpful images, content managers, news writers, and user-interface designers perform page layout, assigning images to concepts. To improve image-retrieval accuracy, a number of issues must be addressed. Readers of businesses reports, newspapers, and social media face the challenge of interpreting large volumes of text in a short amount of time. Students, Teachers, Professors or History enthusiast face the challenge to identify large data in a short amount of time. This can lead to loss of important information. Survey shows that images related to such topics are helpful for the reader to understand and remember the context for a better period of time. There are also people who are not able to take in written matter at a standard pace images sure can boost their grasping ability. This work will help to resolve such cases. The user can easily identify the concept according to the images rather than the text.
\end{abstract}

\section{Keywords}

History, Natural Language Processing

\section{INTRODUCTION}

A picture is worth a thousand words. This proverb reveals the importance of visualization in explaining a concept or word. Suppose someone is introducing the concept of an animal, such as elephant, to a child. If he/she can provide some pictures of elephant to this child in the description, it will be more readily for the child to comprehend the meanings. Since manually finding such appropriate images is time consuming, we aims to develop a system that can automatic generate sets of images to visually interpret a given word. Automatically linking word to image is very helpful for people to rapidly and conveniently acquire knowledge, but it also involves some challenging issues. First, the correctness of linked images is critical; otherwise unrelated images will lead to misunderstanding. Second, since most word has different semantic aspects, the result images should be diverse enough to represent these aspects. Third, representative images should be selected from the image sets to reduce redundancy, that is to say, we should present the compact and visual appealing results to the users. Therefore, an automated word to image translation system should satisfy four requirements. They are: precision, diversity, representativeness of result images, and the friendliness and appealing of interface. The importance of the digital society in various aspects of our lives is undeniable. Allowing people with cognitive disabilities to independently Internet can increase their quality of life, by reducing social isolation. Augmentative and Alternative Communication (AAC) assists people with severe communication disabilities to be more socially active in interpersonal interaction, learning, education, community activities, employment, volunteering, and care management. Picture-based communication systems are a form of AAC technology based on the use of graphics, such as drawings, pictographs, and symbols. Text picturing applications similarly help users' understanding of textual content and can help productivity. Several applications can benefit from text picturing, classroom material, visual summary of reports, advertising, news, and social media.

Text picturing is considered as a human information retrieval task, where a machine reads text input and transcribes that input to a set of visual outputs using information extraction and matching use the techniques. Picture matching requires the semantic match of input text concepts and output image concepts. The knowledge resolution parameter determines the number of pictures for each basic linguistic unit (word/ 
sentence / paragraph). Text picturing systems transform input text into pictures, usually implementing a processing pipeline where text is first processed into basic linguistic units (such as sentences), knowledge is extracted, and pictures are found.

\section{LITRATURE REVIEW}

[1] Francis Ferraro, Nasrin Mostafazadeh, Ting-Hao (Kenneth) Huang, "A Survey of Current Datasets for Vision and Language Research" In this paper, we propose a set of quality metrics for evaluating and analyzing the vision \& language datasets and categorize them accordingly. Our analyses show that the most recent datasets have been using more complex language and more abstract concepts; however, there are different strengths and weaknesses in each.

[2] Christina White Lori Breslow," Exploring Visual Literacy as a Global Competency: An International Study of the Teaching and Learning of Communication

Our larger, comprehensive research study explores the teaching and learning of four communication capabilities: writing, developing and delivering presentations, visual literacy, and participating in teams. This paper focuses specifically on opportunities for undergraduate engineering students to develop visual literacy skills at three universities located in two different countries.

[3] Sharon Oviatt, "Human-Centered Design Meets Cognitive Load Theory: Designing Interfaces that Help People Think"

In this paper, we illustrate different user-centered design principles and specific strategies, as well as their advantages and the manner in which they enhance users' performance. Graphical interfaces still are surprisingly limited in the expressive power and breadth of input capabilities that they support. We describe and evaluate a text-to-pictograph translation system that is used in an online platform for Augmentative and Alternative Communication, which is intended for people who are not able to read and write, but who still want to communicate with the outside world.

[4] Vincent Vandeghinste, Frank Van Eynde, "Translating text into pictographs",

The system looks for the nearest pictographs in the lexicalsemantic database and displays the message into pictographs. In future versions we might consider higher order versions, or more complex approaches towards text normalization, if deemed necessary.

[5] Ayşe Goker, Richard Butterworth, "Expeditions through image jungles: the commercial use of image libraries in an online environment".

Searching for appropriate images as part of a work task is a non-trivial problem. Journalists and Copywriters need to find images that are not only visually appropriate to accompany the documents they are creating, but are acceptably priced and licensed. Further research into time factors is required and the impact that limited time has on information seeking in online journalist environments where large amounts of information must be filtered and evaluated quickly.

\section{MOTIVATION}

For a teenager the subject history is not always the most interesting one as the subject usually contains too many words and is boring to study. Our motivation is to make history more understandable with the help of pictorial representation. The objective is to reduce a reader's subjective information, processing that information and using this by retrieving the most relevant images along with processed input text. A reader can quickly and easily decide if the text information deserves a closer look.

\section{SYSTEM ARCHITECTURE}

The system will make user to understand the critical concept using image as compare to text. For authentication of the user system will provide the security in the form of OTP. Without OTP user cannot register them to the system. The system will be used by the teachers and students. The system will have textual information relating to history Textbook taught to class 9 students on which we will apply stemming porter algorithm and Graphical analysis which will help identify the keywords. These keywords will help extract the relevant images with processed input text.

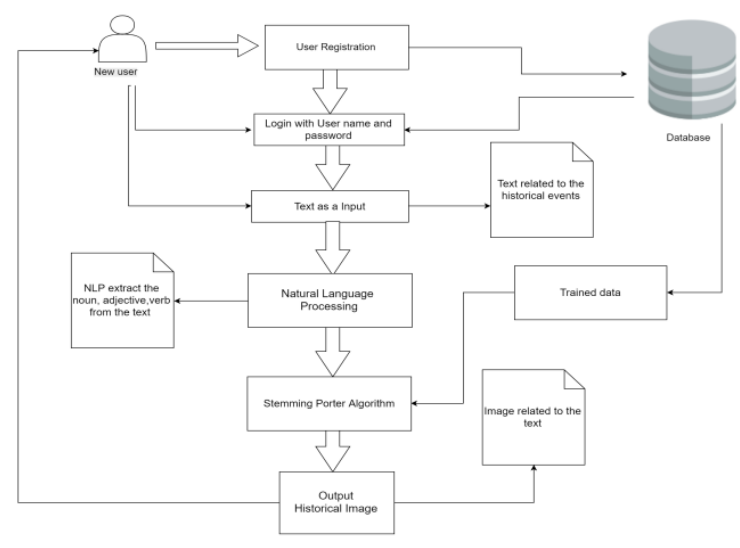

Fig.01 Proposed System

\section{CONCLUSION}

\section{Conclusion:}

IT professionals are familiar with visual interfaces and how they can help user productivity. Text picturing applications similarly help users' understanding of textual content and can help productivity. Several applications can benefit from text picturing, including AAC tools, classroom material, visual summary of reports, advertising, news, and social media. Text picturing remains an active topic of research. There are many opportunities to realize its potential in real-world applications to help with text understanding. Finally, we would like to point out that a related dual problem to the one introduced in this article is the task of automatic image caption generation.22 While deferring detailed discussion of this to others, we note that there is an important role for solutions to the dual problem; that is, the neural-network image-decoding approaches currently found in image-interpreting solutions. These solutions to the dual problem might find their way into solutions for text picturing.

\section{REFERENCES}

[1] S. Oviatt, "Human-Centered Design Meets Cognitive Load Theory: Designing Interfaces that Help People Think," Proc. 14th ACM Int'l Conf. Multimedia (MM), 2006, pp. 871-880.

[2] A. Göker et al., "Expeditions through Image Jungles: The Commercial Use of Image Libraries in an Online Environment," J. Documentation, vol. 72, no. 1, 2016, pp. 5-23.

[3] C. White, L. Breslow, and D. Hastings, "Exploring Visual Literacy as a Global Competency: An International Study of the Teaching and Learning of 
Communication," Proc. 2015 Int'l Conf. Interactive Collaborative Learning (ICL), 2015, pp. 771-778.

[4] B.J. Baars and N.M. Gage, Fundamentals of Cognitive Neuroscience: A Beginner's Guide, Academic Press, 2012.

[5] J. Johnson, Designing with the Mind in Mind: Simple Guide to Understanding User Interface Design Guidelines, Morgan Kaufman, 2010.

[6] V. Vandeghinste, I.S.L. Sevens, and F. Van Eynde, "Translating Text intoPictographs," Natural Language Eng., vol. 23, no. 2, 2017, pp. 217-244.
[7] A.M. Cook and J.M. Polgar, Assistive Technologies: Principles and Practice, Mosby,2015.

[8] M.B. McGrath and J.R. Brown, "Visual Learning for Science and Engineering," IEEE Computer Graphics and Applications, vol. 25, no. 5, 2005, pp. 56-63.

[9] D. Joshi, J.Z. Wang, and J. Li, "The Story Picturing Engine-A System for Automatic Text Illustration," ACM Trans. Multimedia Computing, Communications, and Applications, vol. 2, no. 1, 2006, pp. 68-89.

[10] K. Bontcheva and D. Rout, "Making Sense of Social Media Streams through Semantics: A Survey," Semantic Web, vol. 5, 2014, pp. 373-403. 